\title{
Atomic and electronic structures of a vacancy in amorphous silicon
}

\author{
Andreas Pedersen, ${ }^{1}$ Laurent Pizzagalli $\odot,{ }^{2, *}$ and Hannes Jónsson $\circledast^{1}$ \\ ${ }^{1}$ Faculty of Physical Sciences and Science Institute, University of Iceland, 107 Reykjavík, Iceland \\ ${ }^{2}$ Departement of Physics and Mechanics of Materials,Institut P', CNRS UPR 3346, Université de Poitiers, SP2MI, \\ Boulevard Marie et Pierre Curie, TSA 41123, 86073 Poitiers Cedex 9, France
}

(Received 18 December 2019; accepted 3 February 2020; published 18 February 2020)

\begin{abstract}
Locally, the atomic structure in well-annealed amorphous silicon appears similar to that of crystalline silicon. We address here the question whether a point defect, specifically a vacancy, in amorphous silicon also resembles that in the crystal. From density-functional theory calculations of a large number of nearly defect-free configurations, relaxed after an atom has been removed, we conclude that there is little similarity. The analysis is based on formation energy, relaxation energy, bond lengths, bond angles, Voronoï volume, coordination, atomic charge, and electronic gap states. All these quantities span a large and continuous range in amorphous silicon and while the removal of an atom leads to the formation of one to two bond defects and to a lowering of the local atomic density, the relaxation of the bonding network is highly effective, and the signature of the vacancy generally unlike that of a vacancy in the crystal.
\end{abstract}

DOI: 10.1103/PhysRevB.101.054204

\section{INTRODUCTION}

Vacancy-related defects in crystalline silicon (c-Si) are some of the most studied classes of defects because of the major role of silicon in electronic-based applications. The properties of the simplest defect, the monovacancy, are now well understood and it serves as a standard benchmark for electronic-structure calculation methods and interatomic potential functions. A low Jahn-Teller distortion reduces the natural $T_{d}$ symmetry to $D_{2 d}$, thus breaking the degeneracy of deep levels in the electronic gap [1]. The relaxed defect can be viewed as a hole surrounded by four threefold coordinated atoms as first neighbors. A formation energy of about $3.5-4.4 \mathrm{eV}$ is obtained from electronic-structure calculations [2-4], in good agreement with available measurements [5,6].

Conversely, little is known about the properties of vacancies in the amorphous phase of silicon (a-Si). When it is prepared by ion implantation, thus free of hydrogen, experimental investigations suggest the existence of point defects that are analogous to vacancies in c-Si [7]. In similarly prepared materials, Laaziri et al. found a deficit in coordination, which suggests the presence of vacancy-related defects $[8,9]$. The concentration of these defects is expected to depend on the degree of relaxation. The heat released during annealing processes is compatible with a point-defect annihilation (PDA) mechanism $[10,11]$.

Moreover, there have been some theoretical studies of vacancies in a-Si. In their pioneering work, Kim et al. showed using tight-binding calculations that monovacancy creation was not associated with deep gap states, conversely to what is observed to occur in c-Si [12]. Another investigation revealed that removing one atom from a disordered silicon model leads to either the full recovery of the bond network or the creation of a stable vacancy [13]. Only four configurations

*Laurent.Pizzagalli@univ-poitiers.fr were studied in each of these studies, which questions the general character of these conclusions. A large number of configurations is required to obtain good statistics.

In two recent works, clear improvements were made addressing this aspect and a total of 216 [14] and 1000 configurations [15] were studied. However, atomic interactions were in these studies modeled by either tight-binding [14] or empirical potential functions [15], which are known not to give an accurate description of defects in covalent materials. Another issue relates to the occurrence of vacancies with a negative formation energy, as observed in two previous investigations $[13,15]$. This would suggest that the a-Si model used as a starting configuration is not fully relaxed. There is therefore a need for additional calculations to address the above-mentioned issues.

An interesting additional point concerns the identification of vacancies in the a-Si material. In c-Si, a vacancy is well characterized and leaves several structural and electronic footprints, which can be used for identification. The question is whether such a similar picture could be valid in the disordered phase. Miranda et al. hinted that it might be difficult to identify vacancies using only structural information [13]. This point was later confirmed by Urli et al., who proposed a criterion involving atomic charge and volume. In fact, their tight-binding investigations suggested that atoms characterized by an excess of charge and a large volume are likely to be neighbors to a vacancy [14]. It is important to test the generality of this vacancy criterion with a more accurate description of the atomic interactions and other a-Si models.

In this paper, we describe investigations of the properties of vacancies in a-Si using density-functional theory (DFT) calculations applied to more than 1000 configurations. Five highly optimized a-Si configurations were considered as initial systems, yielding only positive vacancy formation energies. We analyze and discuss the energetics associated with vacancy creation, as well as changes occurring in structure, connectivity, and electronic properties during relaxation. 
TABLE I. Characteristics of several a-Si models, obtained after annealing using DFT/PBE and PDA procedures, starting from a continuous random network (CRN) or from a molecular dynamics liquid quench using the Stillinger-Weber $(\mathrm{SW})$ or Tersoff $\left(\mathrm{T}_{n}\right)$ potentials: Number of atoms $(N)$, number of threefold $\left(\mathrm{C}_{3}\right)$, fourfold $\left(\mathrm{C}_{4}\right)$, and fivefold $\left(\mathrm{C}_{5}\right)$ coordinated atoms, density difference $(\Delta \rho)$, and excess energy $(\Delta e)$ relative to crystalline silicon.

\begin{tabular}{lcccccc}
\hline \hline Type & $N$ & $\mathrm{C}_{3}$ & $\mathrm{C}_{4}$ & $\mathrm{C}_{5}$ & $\Delta \rho$ & $\Delta e[\mathrm{eV} / \mathrm{at}]$. \\
\hline $\mathrm{CRN}$ & 200 & 0 & 200 & 0 & $-2.5 \%$ & 0.148 \\
$\mathrm{SW}$ & 214 & 0 & 212 & 2 & $-1.3 \%$ & 0.147 \\
$\mathrm{~T}_{1}$ & 213 & 0 & 213 & 0 & $-0.9 \%$ & 0.148 \\
$\mathrm{~T}_{2}$ & 209 & 1 & 207 & 1 & $-1.5 \%$ & 0.153 \\
$\mathrm{~T}_{3}$ & 213 & 0 & 213 & 0 & $-0.7 \%$ & 0.156 \\
\hline \hline
\end{tabular}

Finally, we discuss the existence of efficient markers to identify vacancies, as well as the suitability of previously proposed criteria.

\section{MODELS AND NUMERICAL SIMULATIONS}

Calculations were performed in the framework of DFT [16,17], using the VASP package [18]. The Perdew-BurkeErnzerhof functional [19] approximation was used to describe the valence electrons, and the projected augmented wave formalism [20] to describe the core electrons. A planewave cutoff of $18 \mathrm{Ry}$ and a $\Gamma$-point sampling were found to be sufficient to obtain well-converged forces and energies. With these parameters, a c-Si lattice constant of $5.468 \AA$ is obtained. We also checked the suitability of our setup by calculating a vacancy in c-Si. After relaxation, we obtained the expected Jahn-Teller distortion, and a formation energy of $3.556 \mathrm{eV}$ in good agreement with available experimental $[5,6]$ and theoretical [2-4] estimates.

A total of five different models for the initial a-Si configuration were considered. One was built from a continuous random model $(\mathrm{CRN})$ procedure [21], while the others were obtained from a liquid-quench procedure in which atomic interactions were represented by commonly used empirical potential functions [22,23]. All systems have been further optimized regarding energy and density by applying the PDA method combined with forces and energies from the DFT calculations [24], leading to high-quality a-Si models in excellent agreement with available experiments [9,11]. Table I summarizes the main characteristics of the configurations. Even those obtained from the liquid-quench procedure include no or very few coordination defects. All a-Si models are characterized by a density slightly lower than that of $\mathrm{c}-\mathrm{Si}$, in agreement with experiments [25].

A vacancy was created by removing one atom, followed by relaxation using conjugated gradients with a force convergence criterion of $0.01 \mathrm{eV} / \AA^{-1}$. This procedure was carried out for all atoms in all five configurations, yielding 1049 vacancy configurations and unprecedented statistics.

\section{ENERGETICS}

The energy required to create a vacancy in the amorphous material is defined as

$$
E_{f}=E-E_{0} \times \frac{N-1}{N},
$$

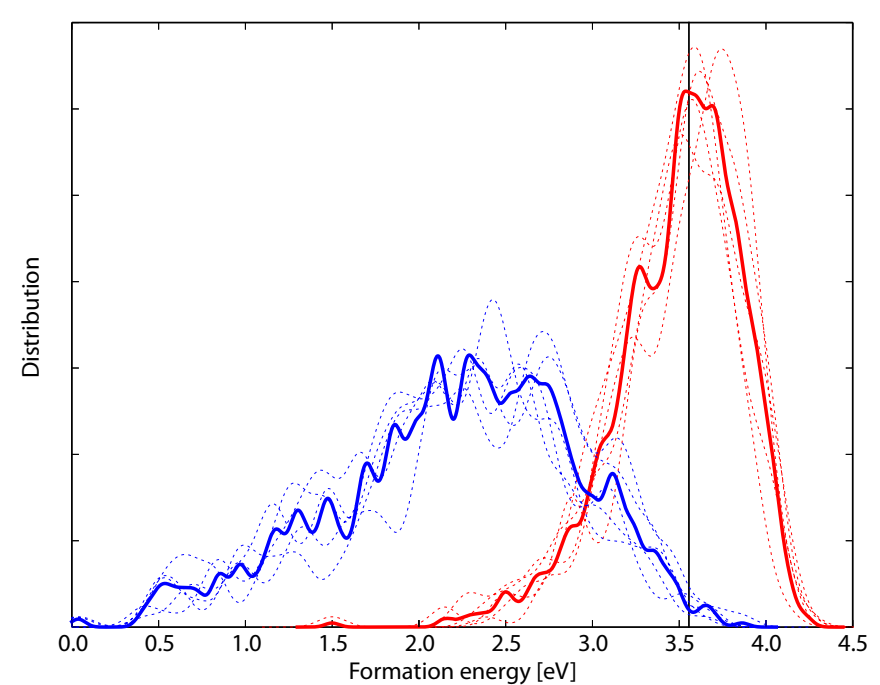

FIG. 1. Distribution of vacancy formation energies, with $\left(E_{f}^{r}\right.$, blue lines) and without ionic relaxation ( $E_{f}^{u}$, red lines) in amorphous silicon. Dashed thin lines show distributions for each of the five configurations (see Table I), while the thick lines show the average. The vertical black line marks the calculated formation energy for a vacancy in c-Si $(3.556 \mathrm{eV})$.

where $E$ is the total energy of the configuration with the vacancy and $E_{0}$ is the total energy of the initial amorphous configuration containing $N$ atoms. Two formation energies can be defined, $E_{f}^{r}$ and $E_{f}^{u}$, depending whether ionic relaxation is taken into account or not. The vacancy formation energy without ionic relaxation $E_{f}^{u}$ is useful to analyze the effect of ionic relaxation in the amorphous network. The effect of ionic relaxation is defined as the positive quantity:

$$
\Delta E=E_{f}^{u}-E_{f}^{r} .
$$

Figure 1 shows the distributions of $E_{f}^{r}$ and $E_{f}^{u}$ for all amorphous configurations. Continuous curves were obtained by Gaussian smoothing of width $0.02 \mathrm{eV}(\sigma)$ for each system, and $0.001 \mathrm{eV}$ for the summed data. The distribution of $E_{f}^{u}$ and $E_{f}^{r}$ is similar for all the configurations. Although the amorphous configurations were initially built using different techniques (CRN or liquid quench), the PDA optimization procedure, which was subsequently applied, yields similar high quality and homogeneous a-Si configurations. The distribution of $E_{f}^{u}$ can be represented by an asymmetric Gaussian ranging from $2 \mathrm{eV}$ to $4.3 \mathrm{eV}$ approximately, and centered on about $3.7 \mathrm{eV}$, similar to the formation energy of a relaxed vacancy in c-Si.

The $E_{f}^{r}$ distribution also exhibits an asymmetric Gaussian shape, and is wider and more flat than for $E_{f}^{u}$ (see Fig. 1). The most probable value for the formation energy of a vacancy in a-Si ranges from $2.1 \mathrm{eV}$ to $2.7 \mathrm{eV}$. Assuming a low migration energy, as is the case for a vacancy in c-Si, these values compare fairly well with recent self-diffusion measurements in a-Si [26]. Another interesting aspect of the distribution is its continuity from slightly above $0 \mathrm{eV}$ to almost $4 \mathrm{eV}$. Since the distribution does not have clear subpeaks, it cannot be used to identify different types of vacancies.

It is interesting to see how low the vacancy formation energy can be (see Fig. 1). This is in contrast to the results 
of the tight-binding calculations of Kim et al. [12], where a minimum value of $1.55 \mathrm{eV}$ was found.

The cause of this difference is not clear, but the different levels of the electronic structure approximation is a possible explanation. Miranda et al. reported a negative vacancy formation energy for one case [13]. Instead, in our work, we found that all vacancies are associated with positive formation energy, a confirmation that our initial amorphous configurations were fully relaxed. Note that the small bump located close to the $0 \mathrm{eV}$ formation energy in Fig. 1 is associated with a positive value.

We also attempted to identify a correlation between the unrelaxed formation energy $E_{f}^{u}$ and the ionic relaxation $\Delta E$, but without success. Configurations with large $E_{f}^{u}$ apparently do not preferably correspond to large relaxation energy.

\section{STRUCTURAL RELAXATION}

In this section, we describe how the disordered network responds when a vacancy is formed. The effect of structural relaxation is analyzed through the variation of several indicators such as bond lengths and angles, and Voronoï volume. We found that in all cases, the largest changes upon vacancy creation are associated with the first-shell neighbors of the atom which is removed to create a vacancy. Thus, network relaxation mostly occurs in the close vicinity of the created vacancies and only a weak long-range relaxation of the network is observed. The small dimensions of our a-Si models might also affect such a relaxation. In the following, we only examine the variation of structural indicators associated with these first-neighbor atoms, defined as atoms within a distance of $2.8 \AA$. This separation corresponds to a well-defined minimum in the radial distribution function of the amorphous network, between the first- and secondshell peaks [24]. Similar definitions were chosen in previous investigations [15,27].

Figure 2 shows how the first neighbors of the removed atom are displaced during the relaxation. Most of these atoms are displaced by $0.2-0.3 \AA$ in the direction toward the newly created vacancy, indicative of an inward relaxation. This is comparable to what occurs in c-Si in similar calculations [28]. An outward relaxation is also obtained in a small proportion of cases.

Additional information is gained by investigating how the length of bonds between first neighbors changes upon vacancy creation (excluding bonds between these atoms and the atom which is removed). Before vacancy creation, the bond length distribution has a well-defined Gaussian-like shape, centered at $2.37 \AA$, as shown in Fig. 3. This value is close to the firstshell-neighbors distance in c-Si $[8,9]$. The full width at half maximum (FWHM) is approximately $0.4 \AA$. The creation of the vacancy induces a sizable lengthening and the most probable value becomes $2.43-2.44 \AA$. The most frequent response of the amorphous network to vacancy formation appears to be partial filling of the empty space left by the removed atom. The Gaussian shape of the distribution is preserved, but the FWHM is now $0.8 \AA$, with extreme values ranging from 2.36 to $2.55 \AA$. This illustrates the diversity in the geometry of final configurations.

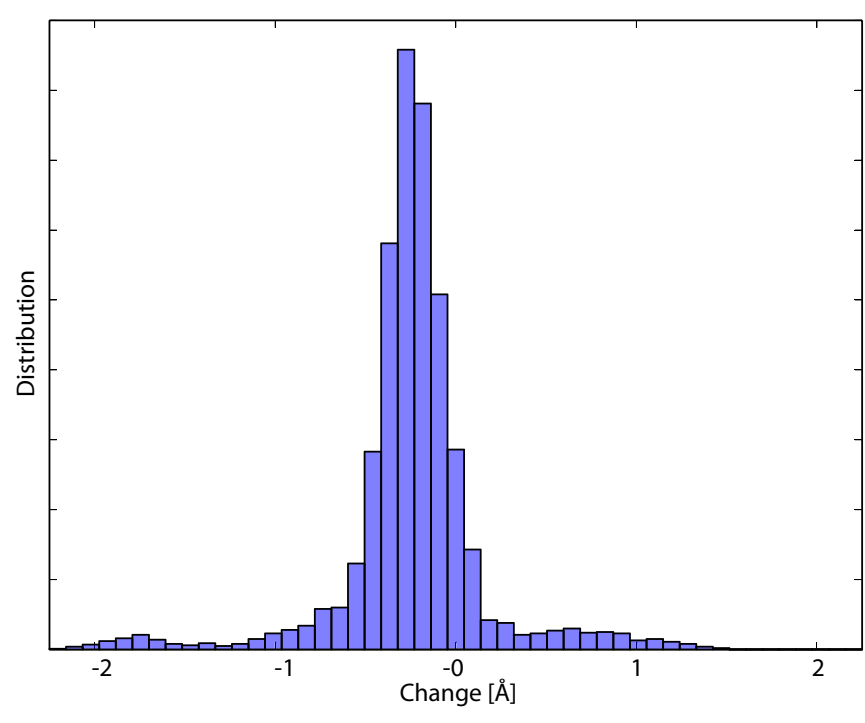

FIG. 2. Distribution of the change in the distance between the site of the atom removed and its first-shell neighbors after vacancy creation. Positive (negative) values indicate an outward (inward) relaxation of the atoms surrounding the vacancy.

To analyze the network relaxation in greater detail, we also examined the changes in the shortest and longest bonds between first neighbors (Fig. 3, bottom). Initially, most of the shortest bonds have a length of $2.3 \AA$, with a quite narrow distribution.

The formation of a vacancy only leads to small changes, with a minor increase of the most probable length to 2.32-2.33 $\AA$, and an almost negligible widening of the distribution. A different situation is found for the longest bonds. Initially, a Gaussian-like distribution centered on lengths of $2.44-2.45 \AA$ and a FWHM of about $0.7 \AA$ is obtained. After vacancy creation, the distribution shape changes and becomes almost flat. All bond lengths ranging from $2.5 \AA$ to the largest considered value of $2.8 \AA$ are possible and equally likely. This indicates that the main relaxation of the network involves these long bonds, which are weak and easy to stretch. Conversely, the shortest bonds are only slightly lengthened.

Changes in angles made between bonds may also provide useful insights about network relaxation. Figure 4 shows the distributions of bond angles before and after vacancy creation for first-shell neighbors of the removed atom. The distribution in the original configurations is characterized by a welldefined maximum at about $108.2^{\circ}$, a value in excellent agreement with experimental determinations $[9,29]$ and indicative of extensive annealing [24]. In the vacancy configurations, the most probable value of the angle between bonds formed by first neighbors is slightly lowered, by about $0.5^{\circ}$. But the most striking difference comes from the width of the distribution. In fact, while bond angles range between $106^{\circ}$ and $110^{\circ}$ in the original configurations, a much wider range from $98^{\circ}$ to $112^{\circ}$ is obtained in the presence of a vacancy, after relaxation. Overall, it seems that the average bond angle for first neighbors tends to become more acute, which can be explained by the inward relaxation previously described.

As for bond lengths, we examined the variations of the widest and narrowest bond angles corresponding to vacancy 

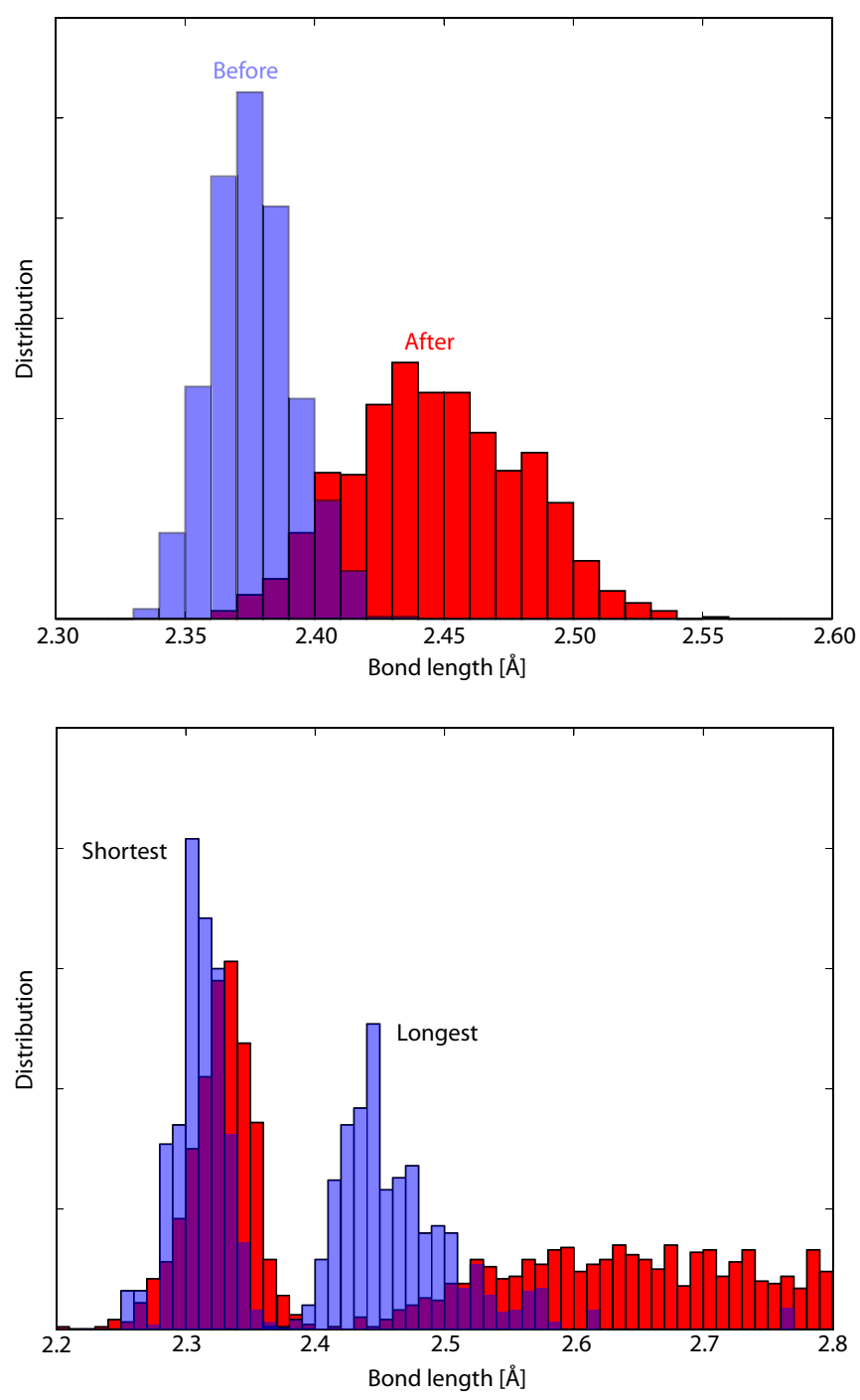

FIG. 3. Top: Average length distribution of the bonds associated to first-shell neighbors of the removed atom (vacancy) before (blue) and after (red) vacancy creation (excluding bonds connecting the removed atom). Bottom: Longest and shortest bond distributions.

first neighbors (Fig. 4). The narrowest angle distribution is initially centered at $92^{\circ}$. When the system has relaxed, the maximum corresponds to a value of $88^{\circ}$, with a minor widening of the distribution. In a few cases, angles as low as $50-60^{\circ}$ can be obtained. The influence of the relaxation is stronger for the widest bond angles. In fact, the initial most probable value of about $123^{\circ}$ increases to about $135^{\circ}$ for the relaxed systems, associated with a sizable widening of the distribution. Final bond angles were found to range from about $100^{\circ}$ to almost $180^{\circ}$. This shows the great diversity of relaxation mechanisms upon vacancy creation in the disordered network.

The atomic volume is another geometrical quantity which can be used to analyze the network relaxation. Although alternative volume definitions are possible [12,13], we considered here the Voronoï volume as in previous investigations [14]. Distributions for vacancy first-shell neighbors with and without vacancy are represented in Fig. 5. Prior to vacancy formation, a symmetric Gaussian-like distribution is obtained, with a maximum occurring at about $20.6 \AA^{3}$ and a FWHM
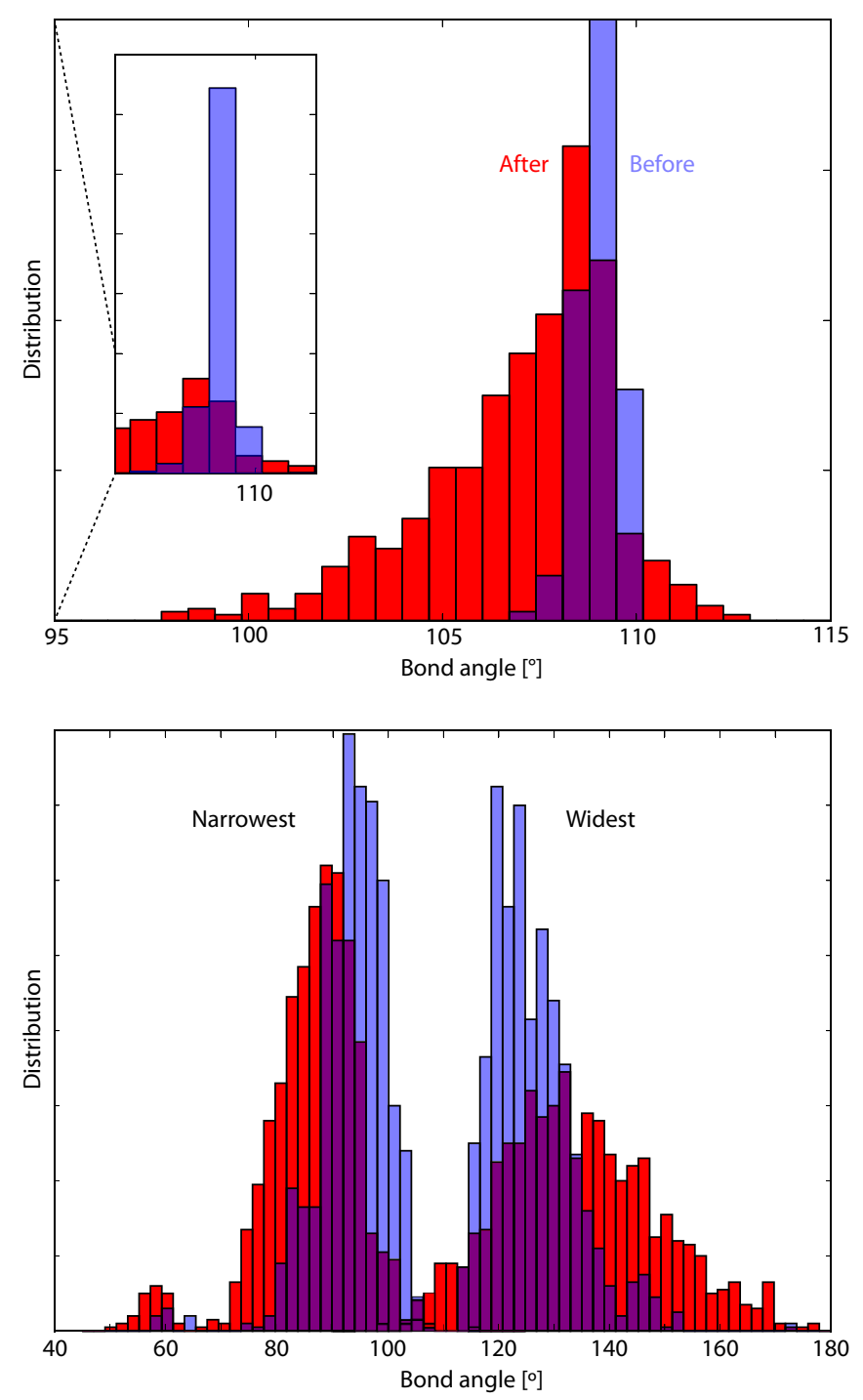

FIG. 4. Top: Average angle distribution between bonds connecting atoms which are first-shell neighbors of the removed atom (vacancy) before (blue) and after (red) vacancy creation. Bonds associated with the removed atom are not considered in the analysis. The inset graph is an enlarged view showing the full height of the distribution. Bottom: Distributions for narrowest and widest angles.

of about $3 \AA^{3}$. This most frequent volume corresponds to amorphous silicon, which is approximately $1 \%$ less dense than the crystal. After vacancy formation, the distribution is shifted toward larger volume with a maximum at $22 \AA^{3}$, becomes wider (FWHM of $5 \AA^{3}$ ) and asymmetric due to an increasing weight of large values. This result is expected, since the decomposition of space according to Voronoï rules is complete and the available volume at the vacancy location is essentially shared by the first neighbors.

\section{NETWORK CONNECTIVITY}

An important aspect of amorphous systems is the connectivity of the bond network. In the case of silicon, the network is formed by fourfold coordinated atoms, with a certain proportion of bond defects which depends on the preparation 


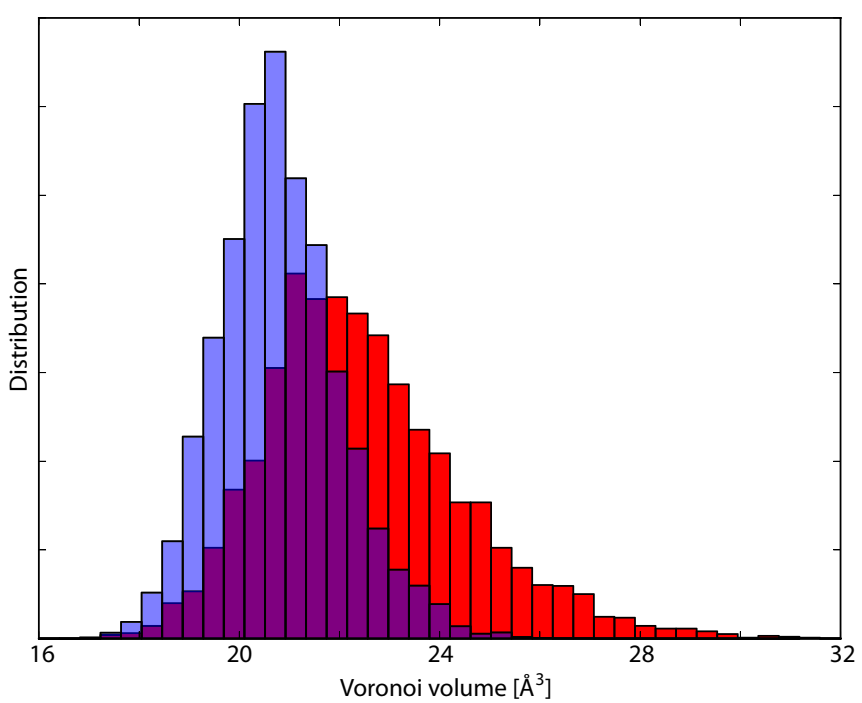

FIG. 5. Voronoï volume distribution associated with vacancy first neighbors before (blue) and after (red) vacancy creation.

method. The creation of vacancies is obviously expected to result in new bond defects in the network. It is therefore interesting to determine to what extent the introduced bond defects can be cured during the relaxation. We determined coordination changes for all atoms in the system, using the same distance criterion as before, i.e., 2.8 $\AA$. Note that a more rigorous determination of atomic coordination would require the analysis of the electronic structure in the vicinity of the vacancy. Unfortunately, this is difficult to automate and would require excessive effort for the large number of configurations considered here.

The original a-Si configurations include no or very few bond defects. Then, the creation of a vacancy by removing an atom mainly results in four fourfold coordinated atoms becoming threefold coordinated, i.e., four $\mathrm{C}_{4} \rightarrow$ four $\mathrm{C}_{3}$, using the notation in Table I. We first compare changes in connectivity in vacancy configurations before and after relaxation. Table II reports the observed changes, sorted according to their rate of occurrences. The most frequent (about 44\%) corresponds to two $\mathrm{C}_{3}$ converted to two $\mathrm{C}_{4}$, i.e., the formation of a bond between two atoms that are first-shell neighbors of the vacancy. This is a partial recovery of the network, leaving only two $\mathrm{C}_{3}$ atoms. The second most frequent event, accounting for one quarter of the total, is surprisingly a full recovery of fourfold coordination, i.e., all four $\mathrm{C}_{3}$ manage to reform bonds. The third most common event is the conversion of three $\mathrm{C}_{3}$ atoms to two $\mathrm{C}_{4}$ and one $\mathrm{C}_{5}$, leaving two bond defects, a dangling bond but also a weak bond. Obtaining overcoordination by removing one atom is counterintuitive at first, and occurs in about $15 \%$ of the cases. Finally, the fourth most common event with an occurrence probability of about $5 \%$ corresponds to four $\mathrm{C}_{3}$, i.e., no connectivity modifications during relaxation. This is the configuration closest to a vacancy in c-Si, characterized by $4 \mathrm{C}_{3}$ atoms. It is surprising how low the probability of this configuration is.

Several other possible situations with low probabilities have also been obtained. One of the most impressive cases is the transformation of the four $\mathrm{C}_{3}$ atoms to four $\mathrm{C}_{5}$ atoms.
TABLE II. List of changes in the number of bonds (defined as pair distance within $2.8 \AA$ ) after a vacancy is created, with their occurrence probability for the five a-Si configurations summarized in Table I. $\mathrm{C}_{n}$ columns report the number of $n$-fold atoms appearing/disappearing during relaxation.

\begin{tabular}{lcccccc}
\hline \hline $\mathrm{C}_{2}$ & $\mathrm{C}_{3}$ & $\mathrm{C}_{4}$ & $\mathrm{C}_{5}$ & $\mathrm{C}_{6}$ & All & CRN \\
\hline 0 & -2 & +2 & 0 & 0 & $43.756 \%$ & $50.5 \%$ \\
0 & -4 & +4 & 0 & 0 & $24.880 \%$ & $29.0 \%$ \\
0 & -3 & +2 & +1 & 0 & $15.062 \%$ & $09.5 \%$ \\
0 & 0 & 0 & 0 & 0 & $04.671 \%$ & $05.0 \%$ \\
0 & -4 & +2 & +2 & 0 & $02.860 \%$ & $03.5 \%$ \\
0 & -1 & +2 & -1 & 0 & $02.764 \%$ & \\
0 & -3 & +4 & -1 & 0 & $02.574 \%$ & \\
0 & -1 & 0 & +1 & 0 & $00.763 \%$ & $01.5 \%$ \\
0 & -3 & 0 & +3 & 0 & $00.477 \%$ & $01.0 \%$ \\
0 & -2 & 0 & +2 & 0 & $00.381 \%$ & \\
0 & -4 & 0 & +4 & 0 & $00.381 \%$ & \\
0 & -4 & +3 & 0 & +1 & $00.286 \%$ & \\
0 & +1 & 0 & -1 & 0 & $00.286 \%$ & \\
0 & -2 & +4 & -2 & 0 & $00.191 \%$ & \\
-1 & -2 & +3 & 0 & 0 & $00.191 \%$ & \\
0 & -5 & +4 & +1 & 0 & $00.191 \%$ & \\
-1 & -3 & +3 & +1 & 0 & $00.095 \%$ & \\
0 & -1 & -2 & +3 & 0 & $00.095 \%$ & \\
0 & -4 & +6 & -2 & 0 & $00.095 \%$ & \\
\hline \hline
\end{tabular}

This was observed four times, and shows that the formation of several weak bond defects can occur when an atom is removed. More generally, we found formation of one or several $\mathrm{C}_{5}$ atoms in about $20 \%$ of the cases. The creation of overcoordinated atoms as a result of introducing a vacancy is unexpected and remarkable. It shows the great diversity of possible network mechanisms for minimizing energy and is in agreement with previous investigations showing that the creation of bond defects can help lower the energy of amorphous silicon [27]. Most of the rare cases in Table II are related to bond defects already present in the a-Si configurations. This is obviously the situation for configurations in which the number of $\mathrm{C}_{5}$ or $\mathrm{C}_{2}$ atoms decreases. For instance, it is interesting to see that the two $\mathrm{C}_{5}$ atoms originally present in the $\mathrm{SW}$ a-Si model (see Table I) can be annihilated, either with a partial (formation of four $\mathrm{C}_{4}$ atoms plus two $\mathrm{C}_{3}$ atoms remaining) or a full recovery (formation of six $\mathrm{C}_{4}$ atoms) of the fourfold coordination of the network, although their original separation is about $10 \AA$. This shows that in a few cases, even the use of local relaxation such as conjugate gradient energy minimization can lead to network rearrangements on a larger scale.

To show that the presence of bond defects initially present in the model has little influence on the statistics, we also report in Table II the results corresponding to the CRN model. One can see that the most frequent relaxation events are the same for all models, with similar occurrence probabilities.

Finally, Fig. 6 shows the proportion of $\mathrm{C}_{3}$ and $\mathrm{C}_{5}$ atoms after vacancy creation and relaxation, compared to the initial a-Si configurations. It is clear that in the majority of the cases, overcoordinated $\mathrm{C}_{5}$ atoms do not form. Regarding undercoordinated $\mathrm{C}_{3}$ atoms, i.e., dangling bonds, the situation is more complex. The most likely outcome is the creation 

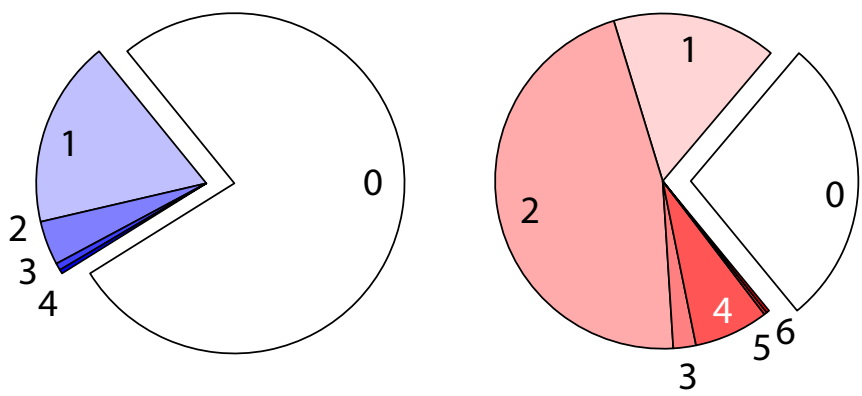

FIG. 6. Proportion of configurations, depending on the number of over-coordinated $\mathrm{C}_{5}$ (left pie chart) and undercoordinated $\mathrm{C}_{3}$ (right pie chart) atoms, resulting from the creation of a vacancy followed by relaxation, compared to initial configurations. For instance, in most of cases, no additional $\mathrm{C}_{5}$ atoms are created (left, white slice 0 ). Also, the most frequent result is the formation of two $\mathrm{C}_{3}$ atoms (right, light red slice 2).

of two dangling bonds, followed by the full recovery of the network (no created $\mathrm{C}_{3}$ ). An examination of the pie chart in Fig. 6 reveals that the formation of several dangling bonds is quite unlikely. An even number of defects is favored over an odd one, since the formation or breaking of a single bond necessarily involves two atoms.

\section{ELECTRONIC STRUCTURE}

DFT calculations make it possible to estimate the electronic structure of the material. Electronic states, located in the band gap of a-Si, are typically associated with dangling or weak bonds. We first investigate the relation between the formation of a vacancy in the amorphous network and the appearance of states in the gap. Compared to what can occur in $\mathrm{c}-\mathrm{Si}$, it is difficult to unambiguously discriminate between states corresponding to regions with structural defects and "normal" states of the amorphous material, even by analyzing the spatial localization of all states characterized by energies in the vicinity of the gap. Here, because of the large number of vacancy configurations, we simply analyzed the number of states appearing in the band gap after vacancy formation. We define such states to have an orbital energy at least $0.25 \mathrm{eV}$ above the valence band edge and $0.25 \mathrm{eV}$ below the conduction band edge.

Table III reports the number of vacancy configurations as a function of the number of new gap states. In most cases, the creation of a vacancy leads to one or two states appearing in the gap. Less frequent situations are three gap states, and no gap states at all. Finally, formation of vacancies with four gap states appears to be rather unlikely, about $1 \%$ of the cases.

TABLE III. Number of cases where none or one to four electronic states appear in the gap when an atom is removed and the configuration relaxed.

\begin{tabular}{lccccc}
\hline \hline Nr of gap states & 0 & 1 & 2 & 3 & 4 \\
\hline Nr of events & 75 & 318 & 484 & 164 & 8 \\
Percentages & $7 \%$ & $30 \%$ & $46 \%$ & $16 \%$ & $1 \%$ \\
\hline \hline
\end{tabular}

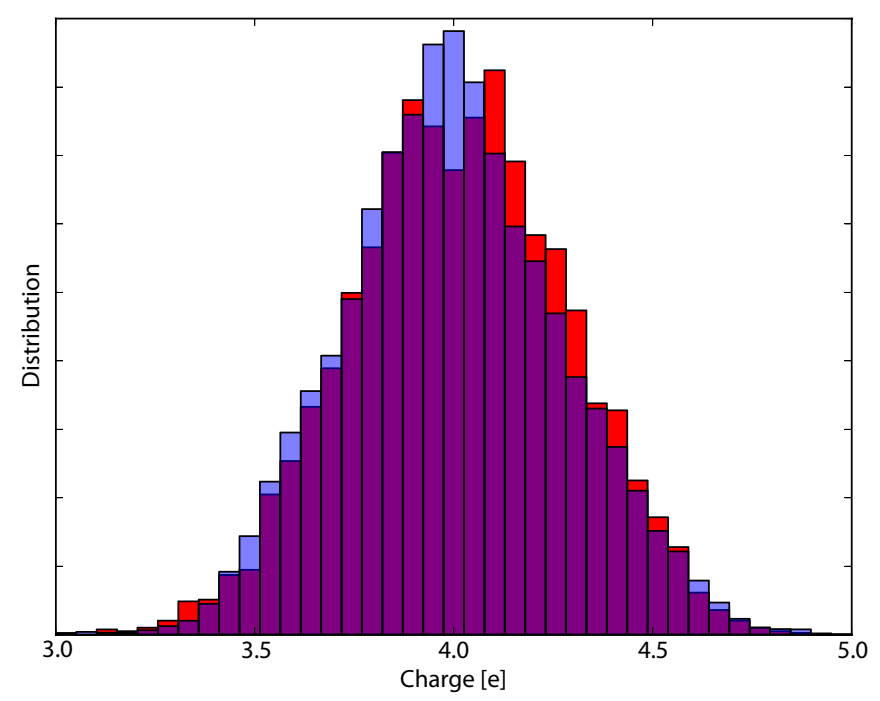

FIG. 7. Charges for atoms, which are first-shell neighbors of the vacancy, computed using a Bader analysis before (blue) and after (red) vacancy creation.

Overall, we can state that the formation of a vacancy in a-Si is usually associated with the generation of states located in the band gap.

We also examined how the creation of a vacancy influences the charge on the atoms belonging to the first-shell of neighbors of the vacancy. The charge distributions, computed following a Bader charge analysis [30,31], are shown in Fig. 7. Before a vacancy is created in the system, a Gaussian-like distribution is obtained with the expected maximum at $4 \mathrm{e}^{-}$. Surprisingly, little changes in the distribution are observed after creation. However, the maximum is now less well defined, with a seemingly double peak structure at 3.9 and 4.1 $\mathrm{e}^{-}$, and there is now a minor asymmetry toward an excess of charge. Nevertheless, the overall initial Gaussian structure is preserved.

\section{DISCUSSION}

The analysis of our data, extracted from our large set of configurations, confirms that it is not possible to firmly identify a vacancy in a-Si on the basis of structural quantities. We especially studied the structural changes for atoms in the closest vicinity of the created vacancy, where the changes are most pronounced. Most of the time, we found an inward relaxation, with small changes in short bonds and a significant stretching of the longest bonds. This is in agreement with Voronoï volume calculations, which indicate an increase of the volume for these atoms. Besides, the analysis of bond angles revealed an increase of the angular dispersion upon vacancy creation. Considering all these indications, it would be tempting to conclude that a cluster of atoms characterized by larger Voronoï volumes, and higher disorder in bond angles, should contain a vacancy. Unfortunately, it is not that simple since we also find many configurations for which the formation of a vacancy is not associated with volume increase or a larger angular disorder. Thus, our investigations confirm that a vacancy in a-Si cannot be identified on the sole basis of structural properties. 


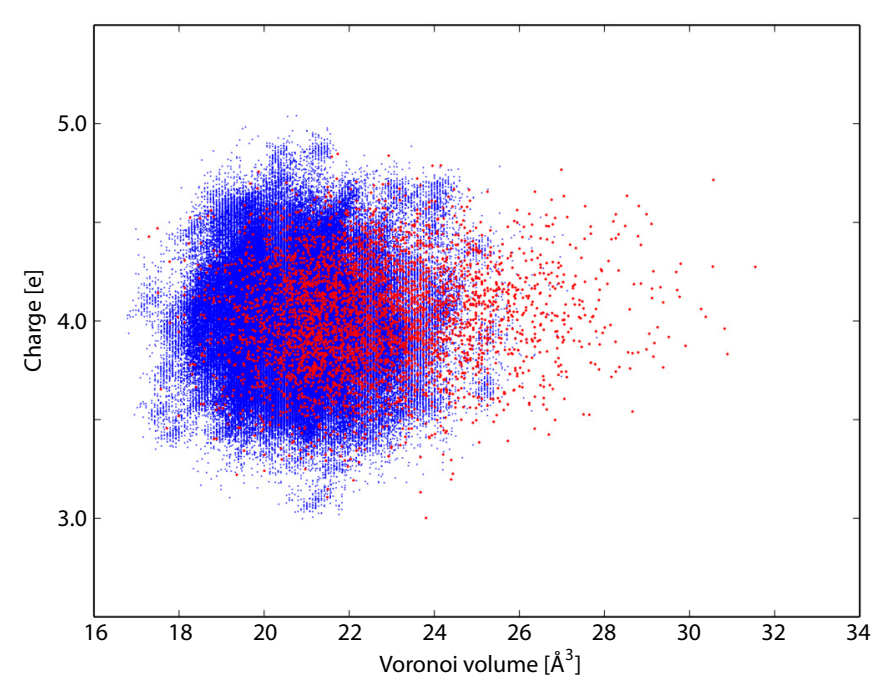

FIG. 8. Charge-volume correlation for all investigated relaxed vacancy configurations. Red dots represent data for atoms which are first-shell neighbors of the vacancy while blue dots represent the remaining atoms.

We also investigated charge variations, as well as the number of states appearing in the gap. The former relates to a characterization proposed by Urli et al. involving correlation between volume and charges on atoms in the vicinity of a vacancy [14]. An increase in volume is supposedly correlated with a charge increase of about $0.15 \mathrm{e}^{-}$on average. This would lead to two sets of atoms with distinct properties. Our results, however, do not show such a clear correlation. We find no significant differences in charge on atoms neighboring the vacancy. The charge-column plot shown in the Fig. 8 exhibits no correlation. While we have no definitive explanation for this difference from the results of Urli et al. [14], it is likely because of the different levels of electronic structure theory employed.

Regarding states in the band gap, it seems that vacancy formation is usually (but not always) associated with the apparition of states in the gap. However, since there were already a few states present in the gap for our initial vacancy-free configurations, it is not possible to draw definitive conclusions on this issue.

The most striking outcome from our investigations is the changes in coordination during the relaxation of the created vacancy. Although one should be aware that a coordination criterion based only on distance between atoms has some limitations, it appears that a great diversity of final configurations can be obtained. Those cover all conceivable situations, from the creation of several overcoordinated defects to the stabilization of the four threefold coordinated atoms as for the c-Si vacancy. In addition, in about one quarter of the investigated cases, the full recovery to a network with no bond defects was obtained. This should be put in context with the distribution of formation energies or relaxation energies, which also span a large range of values. Attempts to find a correlation between these energies and the network relaxation or the final connectivity were unsuccessful. In fact, it is possible to find cases for which a low formation energy is associated with a configuration similar to the vacancy in c-Si, i.e., four $\mathrm{C}_{3}$, and others for which the full recovery of the network is obtained at the cost of energy in the upper range of the computed values.

Our investigations show that most of the computed properties change after the creation of a vacancy in the amorphous configuration. But the distribution of possible values for the final configuration is continuous and homogeneous in all cases, and it always overlaps with the distribution from vacancy-free systems. In fact, we did not find a quantity for which there are two well-defined and separated sets of values. In other words, none of the quantities we investigated can be used to define a criterion for vacancy identification. Based on this statement, the obvious question is whether one can consider that a vacancy in amorphous silicon really exists. By analogy with the crystalline phase, it is tempting to consider that a vacancy is located at the center of four $\mathrm{C}_{3}$ atoms. Using this definition and a similar criterion for connectivity, Joly et al. found that only $45 \%$ of the created vacancies survived the initial structural relaxation [15]. In our work, using the same vacancy definition, only $5 \%$ were found to be stable following the structural relaxation, the difference being probably due to the superior ability of first-principles calculations to break/create bonds compared to empirical potentials. Joly et al. also performed long-range relaxation using the kinetic activation-relaxation k-ART technique, during which almost all remaining vacancies disappeared. Even if such calculations are out of the scope of the present paper, it is very likely that a similar result would be obtained for our remaining $5 \%$ configurations. The conclusion is therefore that vacancies similar to those of the crystal may exist in a-Si, but solely as rare and temporary excited states. Interestingly, similar questions have been raised for other amorphous materials such as $\mathrm{Al}_{2} \mathrm{O}_{3}$ and $\mathrm{HfO}_{2}[32,33]$.

To summarize, our investigations on a large set of configurations suggest that removing one atom in an amorphous system can lead to two cases. The first one is the full recovery of the network, thus with no coordination defects, with a locally lower density and probably at the expense of a larger local stress. The second one is the formation of one to two coordination defects, which could be dangling bonds (attached to $\mathrm{C}_{3}$ atoms) or weak bonds (associated with $\mathrm{C}_{5}$ atoms). Dangling bonds are favored over weak bonds, mainly because here we remove atoms from the network. It is likely that a procedure based on the insertion of additional atoms in the network would favor the opposite case.

Finally, it is worth mentioning that the structure of amorphous materials depends on how samples are prepared, in both experiments and simulations. The properties of a structural defect such as a vacancy could then be similarly dependent. In this study, we used different models, which aim at representing well-relaxed and annealed a-Si materials [24]. Further investigations are probably needed to test whether our findings and conclusions remain valid for different a-Si models.

\section{CONCLUSION}

The properties of created vacancies in amorphous silicon have been investigated using DFT. A large set of more than 1000 configurations has been considered in this paper, allowing for unprecedented statistics. Highly optimized a-Si models 
were considered as initial systems, yielding only positive vacancy formation energies. We determined and analyzed the distributions of formation and relaxation energies, bond lengths and angles, Voronoï volumes, coordination, atomic charge, and creation of gap states. We could not identify markers characteristic of a created vacancy, since possible values for all these quantities span over a large and continuous range. Configurations similar to the vacancy in crystalline silicon appear to be unlikely, except as temporary excited states. Finally, the most probable formed defects in a-Si following the removal of one atom are either a lowering of local density as the bond network fully recovers, or the formation of one to two bond defects. It would be interesting to investigate the stability of these defects by performing long-range relaxation dynamics, using, for example, the adaptive kinetic Monte Carlo method for long timescale simulations [34].

\section{ACKNOWLEDGMENTS}

We would like to thank G. Barkema for providing us the initial CRN model. The computations were carried out using the Nordic High Performance Computing (NHPC) facility in Iceland. We acknowledge financial support from the Icelandic Research Fund and the French foreign ministry, within the Icelandic-French project called Jules Verne. A.P. also acknowledges financial support from the Région PoitouCharentes for his stay at the Prime Institute.
[1] M. J. Puska, S. Pöykkö, M. Pesola, and R. M. Nieminen, Phys. Rev. B 58, 1318 (1998).

[2] F. El-Mellouhi, N. Mousseau, and P. Ordejón, Phys. Rev. B 70, 205202 (2004).

[3] D. Caliste and P. Pochet, Phys. Rev. Lett. 97, 135901 (2006).

[4] M. Kaltak, J. Klimeš, and G. Kresse, Phys. Rev. B 90, 054115 (2014).

[5] S. Dannefaer, P. Mascher, and D. Kerr, Phys. Rev. Lett. 56, 2195 (1986).

[6] T. Südkamp and H. Bracht, Phys. Rev. B 94, 125208 (2016).

[7] G. N. van den Hoven, Z. N. Liang, L. Niesen, and J. S. Custer, Phys. Rev. Lett. 68, 3714 (1992).

[8] K. Laaziri, S. Kycia, S. Roorda, M. Chicoine, J. L. Robertson, J. Wang, and S. C. Moss, Phys. Rev. Lett. 82, 3460 (1999).

[9] K. Laaziri, S. Kycia, S. Roorda, M. Chicoine, J. L. Robertson, J. Wang, and S. C. Moss, Phys. Rev. B 60, 13520 (1999).

[10] S. Roorda, S. Doorn, W. C. Sinke, P. M. L. O. Scholte, and E. van Loenen, Phys. Rev. Lett. 62, 1880 (1989).

[11] S. Roorda, W. C. Sinke, J. M. Poate, D. C. Jacobson, S. Dierker, B. S. Dennis, D. J. Eaglesham, F. Spaepen, and P. Fuoss, Phys. Rev. B 44, 3702 (1991).

[12] E. Kim, Y.-H. Lee, C. Chen, and T. Pang, Phys. Rev. B 59, 2713 (1999).

[13] C. Miranda, A. Antonelli, A. da Silva, and A. Fazzio, J. NonCryst. Solids 338-340, 400 (2004).

[14] X. Urli, C.L. Dias, L. J. Lewis, and S. Roorda, Phys. Rev. B 77, 155204 (2008).

[15] J.-F. Joly, L. K. Béland, P. Brommer, and N. Mousseau, Phys. Rev. B 87, 144204 (2013).

[16] P. Hohenberg and W. Kohn, Phys. Rev. 136, B864 (1964).
[17] W. Kohn and L. J. Sham, Phys. Rev. 140, A1133 (1965).

[18] G. Kresse and D. Joubert, Phys. Rev. B 59, 1758 (1999).

[19] J. P. Perdew, K. Burke, and M. Ernzerhof, Phys. Rev. Lett. 77, 3865 (1996).

[20] P. E. Blöchl, Phys. Rev. B 50, 17953 (1994).

[21] G. T. Barkema and N. Mousseau, Phys. Rev. B 62, 4985 (2000).

[22] F. H. Stillinger and T. A. Weber, Phys. Rev. B 31, 5262 (1985).

[23] J. Tersoff, Phys. Rev. B 38, 9902 (1988).

[24] A. Pedersen, L. Pizzagalli, and H. Jónsson, New J. Phys. 19, 063018 (2017).

[25] J. S. Custer, M. O. Thompson, D. C. Jacobson, J. M. Poate, S. Roorda, W. C. Sinke, and F. Spaepen, Appl. Phys. Lett. 64, 437 (1994).

[26] J. Kirschbaum, T. Teuber, A. Donner, M. Radek, D. Bougeard, R. Böttger, J. L. Hansen, A. N. Larsen, M. Posselt, and H. Bracht, Phys. Rev. Lett. 120, 225902 (2018).

[27] N. Bernstein, J. L. Feldman, and M. Fornari, Phys. Rev. B 74, 205202 (2006).

[28] J. Lento and R. M. Nieminen, J. Phys.: Condens. Matter 15, 4387 (2003).

[29] J. Fortner and J. S. Lannin, Phys. Rev. B 39, 5527(R) (1989).

[30] R. Bader, Atoms in Molecules: A Quantum Theory (Oxford University Press, New York, 1990).

[31] G. Henkelman, A. Arnaldsson, and H. Jónsson, Comput. Mater. Sci. 36, 354 (2006).

[32] P. Broqvist and A. Pasquarello, Microelectron. Eng. 84, 2022 (2007).

[33] Z. Guo, F. Ambrosio, and A. Pasquarello, Appl. Phys. Lett. 109, 062903 (2016).

[34] G. Henkelman and H. Jónsson, J. Chem. Phys. 115, 9657 (2001). 